\title{
Determination of ground and excited state dipole moments via electronic Stark spectroscopy: 5-methoxyindole
}

Cite as: J. Chem. Phys. 144, 044201 (2016); https://doi.org/10.1063/1.4940689

Submitted: 11 November 2015 . Accepted: 08 January 2016 . Published Online: 28 January 2016

Josefin Wilke, Martin Wilke, (D) W. Leo Meerts, and Michael Schmitt
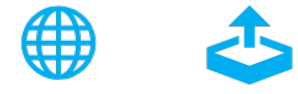

\section{ARTICLES YOU MAY BE INTERESTED IN}

Density-functional thermochemistry. III. The role of exact exchange

The Journal of Chemical Physics 98, 5648 (1993); https://doi.org/10.1063/1.464913

Natural transition orbitals

The Journal of Chemical Physics 118, 4775 (2003); https://doi.org/10.1063/1.1558471

Adiabatic time-dependent density functional methods for excited state properties

The Journal of Chemical Physics 117, 7433 (2002); https://doi.org/10.1063/1.1508368

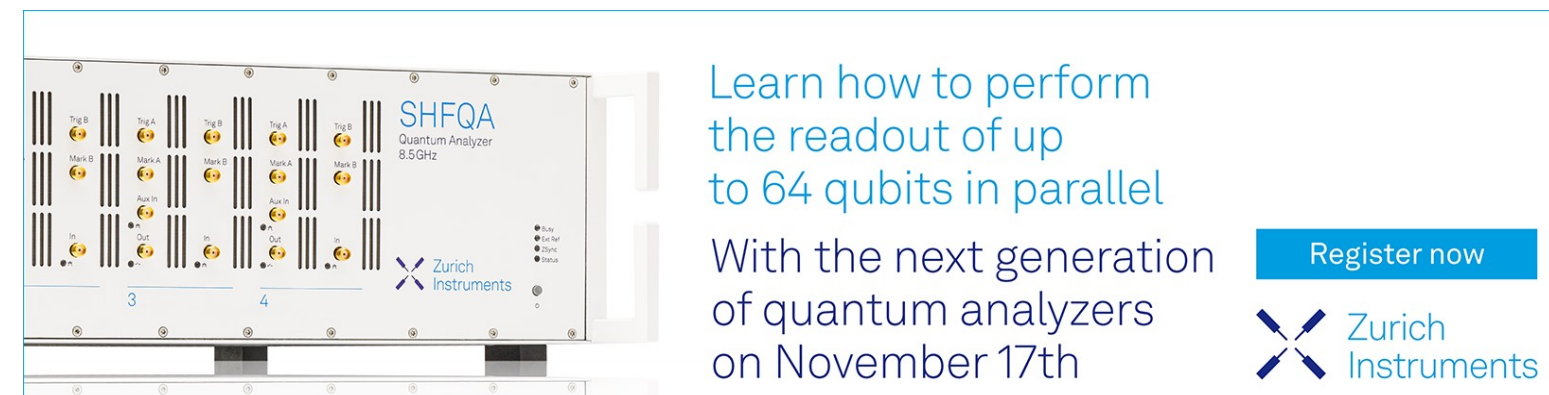




\title{
Determination of ground and excited state dipole moments via electronic Stark spectroscopy: 5-methoxyindole
}

\author{
Josefin Wilke, ${ }^{1}$ Martin Wilke, ${ }^{1}$ W. Leo Meerts, ${ }^{2, a)}$ and Michael Schmitt ${ }^{1, b)}$ \\ ${ }^{1}$ Institut für Physikalische Chemie, Heinrich-Heine-Universität, D-40225 Düsseldorf, Germany \\ ${ }^{2}$ Institute for Molecules and Materials, Radboud University, NL-6525 AS Nijmegen, The Netherlands
}

(Received 11 November 2015; accepted 8 January 2016; published online 28 January 2016)

\begin{abstract}
The dipole moments of the ground and lowest electronically excited singlet state of 5-methoxyindole have been determined by means of optical Stark spectroscopy in a molecular beam. The resulting spectra arise from a superposition of different field configurations, one with the static electric field almost parallel to the polarization of the exciting laser radiation, the other nearly perpendicular. Each field configuration leads to different intensities in the rovibronic spectrum. With an automated evolutionary algorithm approach, the spectra can be fit and the ratio of both field configurations can be determined. A simultaneous fit of two spectra with both field configurations improved the precision of the dipole moment determination by a factor of two. We find a reduction of the absolute dipole moment from 1.59(3) D to 1.14(6) D upon electronic excitation to the lowest electronically excited singlet state. At the same time, the dipole moment orientation rotates by $54^{\circ}$ showing the importance of the determination of the dipole moment components. The dipole moment in the electronic ground state can approximately be obtained from a vector addition of the indole and the methoxy group dipole moments. However, in the electronically excited state, vector addition completely fails to describe the observed dipole moment. Several reasons for this behavior are discussed. (C) 2016 AIP Publishing LLC. [http://dx.doi.org/10.1063/1.4940689]
\end{abstract}

\section{INTRODUCTION}

In the present publication, we will show optical Stark spectra in a molecular beam environment employing two different field configurations simultaneously. For the static electric field being $100 \%$ parallel or perpendicular to the polarization of the exciting laser radiation, different selection rules exist. By rotating the polarization of the laser light, a superposition of transitions from both selection rules occurs. Spectra with a constant ratio of both field configurations (80\%:20\% and 20\%:80\%) are fit simultaneously to a common set of dipole moments in the electronic ground and excited states, respectively.

While for the electronic ground state the additivity of bond dipole moments is a well-known rule with wide applicability in organic chemistry for molecules in solution, this concept has rarely been tested for isolated molecules or for molecules in electronically excited states. The group of Pratt pioneered this application using electronic Stark spectroscopy. ${ }^{1}$

The dipole moments of electronically excited states may differ considerably from those of the ground state, as a consequence of the altered electron distribution upon excitation. This change of the dipole moment upon electronic excitation has a large influence on solvation dynamics, which is largely governed by the interactions of solute and solvent dipole moments. Also, the conformations of flexible biomolecules like peptides depend on the relative

\footnotetext{
${ }^{a)}$ E-mail: leo.meerts@science.ru.nl

b)E-mail: mschmitt@uni-duesseldorf.de
}

orientations of the backbone dipole moments, the $\alpha$-helix being the consequence of aligned microscopic dipole moments of the peptide bonds. ${ }^{2,3}$ The conformations might be subjected to geometry changes upon the changed dipolar interactions between the different moieties after electronic excitation.

At the same time, the size of the dipole moment can be used to label electronically excited states. For cata-condensed aromatic hydrocarbons, the two lowest electronically excited states are called $\mathrm{L}_{a}$ and $\mathrm{L}_{b}$ states in the nomenclature of Platt. ${ }^{4}$ The labeling scheme has later been extended to indoles by Weber. ${ }^{5}$ One has to keep in mind however that for molecules without two-fold rotation axis along the long inertial axis of the molecule, the meaningfulness of this nomenclature is doubtful. For molecules with the required symmetry, the transition moment of $L_{a}$ goes through the atoms and for $L_{b}$ it runs through the bonds. The close-lying $\mathrm{L}_{a}$ and $\mathrm{L}_{b}$ states in indoles have very different dipole moments, the $\mathrm{L}_{a}$ state dipole moment being much larger than that of the $\mathrm{L}_{b}$ state. ${ }^{6,7}$ Thus, both states have very different stabilizations in polar surroundings. The different dipole moments can be used to distinguish easily between the different electronic states. Of course, this applies to all electronically excited states that differ in dipole moment. The determination of the permanent dipole orientation is based on frequency shifts in an electric field, while the transition dipole moment is evaluated from relative intensities in the rovibronic spectrum, which have a larger experimental uncertainties than the frequencies. Therefore, the angle of the permanent dipole moment can be determined more precisely than that of the transition dipole moment, in cases, in which the electronic nature of the excited state is still unknown. 
Historically, excited-state dipole moments (or more precisely, their changes upon electronic excitation) have been determined from electrooptical absorption measurements ${ }^{8}$ or from solvatochromic shifts in solvents of different static dielectric constants. ${ }^{9,10}$ This method of using solvent shifts engender criticism by Lombardi, ${ }^{11,12}$ who compared so determined excited state dipole moment changes with those from high resolution gas phase Stark effect measurements. In some cases, the condensed phase values exceeded the more precise gas phase values by an order of magnitude. The reason for this strong discrepancy is the approximative nature of the field induced perturbations in case of the strong fields, exerted by the solvents. This strong field couples especially energetically close-lying electronically excited states, leading to an effective dipole moment, which is a combination of the excited state dipole moments of the coupled states as well as the transition moment between these two states. ${ }^{12}$ This effect can be viewed as a field induced dipole moment borrowing from the state with the larger dipole moment. Especially for substituted indoles, for which the energetic distance between the lowest electronically excited singlet states is small, this perturbation plays a large role. The use of thermochromic shifts of fluorescence spectra for the determination of $\mathrm{S}_{1}$-state dipole moments ${ }^{13,14}$ yields more reliable values than the aforementioned methods, because artificial effects by the use of different solvents are excluded.

The most reliable values for dipole moments of ground and electronically excited states are obtained from gas phase electronic Stark experiments, since the dependence of the frequency shift of individual rovibronic lines from the electric field strength yields immediately the dipole moment in ground and excited states. Moreover, not only the absolute value of the total dipole moment can be determined but also the unsigned Cartesian components of the dipole moment in both electronic states. Along with quantum chemical computations, they can be used to determine the absolute orientation of the dipole moment. This is especially important in cases in which the orientation of the dipole moment changes upon electronic excitation. Okruss and Hese ${ }^{15}$ and later Pratt and co-workers ${ }^{16}$ used the Stark effect in rotationally resolved electronic spectra of large polyatomic molecules to determine the dipole moments in the ground and the lowest electronically excited singlet states. Apart from these spectroscopic techniques, a time domain approach to excited state dipole moments in the gas phase is used in coherent beat techniques with a Stark field, pioneered in the groups of Zare, ${ }^{17}$ Huber, ${ }^{18}$ and Tanaka. ${ }^{19}$

The methoxy substituent in 5-position of 5-methoxyindole (5MOI) leads to a large redshift of the ${ }^{1} L_{b}$ state relative to that of indole and the ${ }^{1} L_{a} /{ }^{1} L_{b}$ gap increases to about $3000 \mathrm{~cm}^{-1}$ as shown by Martinaud and Kadiri. ${ }^{20}$ Additionally to the energy gap between the lowest singlet states, methoxy substituents may change the angle $\theta_{a b}$ between the ${ }^{1} L_{a}$ and ${ }^{1} L_{b}$ transition dipole moment vectors, as shown by Eftink et al. ${ }^{21}$ $\theta_{a b}$ is $92^{\circ}$ for the unsubstituted indole 22 and $85^{\circ}$ for 5-methoxyindole. ${ }^{23}$

Resonant two-photon ionization (R2PI) spectra of jetcooled 5MOI have been reported by Hager et al. ${ }^{24}$ up to $1200 \mathrm{~cm}^{-1}$ above the electronic origin and excited state lifetimes have been determined for various vibronic bands. R2PI spectra of 5MOI and of 1:1 and 1:2 adducts with water, methanol, and triethylamine were presented by Huang and Sulkes. ${ }^{25}$ The rotational constants of 5MOI in the electronic ground state and of several vibronic bands of the excited ${ }^{1} L_{b}$ state have been determined by Brand et al. ${ }^{26}$ which proved that all observed bands belong to the anti-methoxy conformer. Florio et al. ${ }^{27}$ showed that all bands in the laser induced fluorescence (LIF) spectrum of 5MOI arise from a single ground state level, which they assigned to the anti-methoxy conformer (see Figure 2).

In what follows, we will present an analysis of the ground and excited state dipole moments of 5MOI by a fit of the dipole moment components to electronic Stark spectra with a constant ratio of two different field configurations.

\section{THEORETICAL METHODS}

\section{A. Ab initio calculations}

The equilibrium geometries of the electronic ground and the lowest excited singlet states were optimized using the approximate coupled cluster singles and doubles model (CC2) employing the resolution-of-the-identity approximation (RI), ${ }^{28-30}$ employing Dunning's correlation consistent polarized valence triple zeta (cc-pVTZ) from the TuRBomole library. ${ }^{31,32}$ Vibrational frequencies and zero-point corrections to the adiabatic excitation energies have been obtained from numerical second derivatives using the NumForce script ${ }^{33}$ implemented in the Turbomole program suite. ${ }^{34}$ The transition state for the interchange of the syn- and anti-conformers was optimized using the Trust Radius Image Minimization (TRIM) algorithm, ${ }^{35}$ implemented in the TuRbomole package, at the CC2/cc-pVTZ level of theory. A natural bond orbital (NBO) analysis ${ }^{36}$ has been performed at the $\mathrm{CC} 2$ optimized geometries using the wave functions from the $\mathrm{CC} 2$ calculations as implemented in the TuRBomole package. ${ }^{34}$

\section{B. Fitting of the rotationally resolved electronic spectra}

The rotationally resolved electronic spectra are fit using evolutionary strategies (ES), described in detail in Refs. 37-40. For the fits of the Stark spectra, we used the covariance matrix adaptation (CMA)-ES algorithm. In this variant, mutations are adapted via a CMA mechanism. The CMA-ES does not leave the choice of strategy parameters open to the user, only the population size can be set. Finding good strategy parameters is considered to be part of the algorithm design. ${ }^{40}$

The spectra with different field configurations were fit simultaneously to a common set of dipole moment components in both electronic states. The intensities of the rotational lines are calculated from the eigenvectors of the Stark Hamiltonian and the direction cosine matrix elements. The static electric field mixes the rovobronic eigenstates, so that $J, K_{a}$, and $K_{c}$ are no good quantum numbers any more. For the static electric field parallel to the polarization of the exciting laser radiation, $M$ is the only remaining good quantum number, and $\Delta M=0$ selection rules hold, while a 
static electric field perpendicular to the polarization of the laser radiation leads to $\Delta M= \pm 1$ selection rules. In principle, the static electric field can also mix torsional eigenstates, which are due to hindered internal motions. Since the barrier to threefold rotation of the methoxy group is so high that with our resolution no torsional splitting for the origin band is observed, a rigid rotor Stark Hamiltonian can be employed. The original source code of David Borst from David Pratt's group in Pittsburgh, in which the Stark contributions to the asymmetric rotor Hamiltonian were implemented, ${ }^{16,41}$ was modified in order to run the program in multi-processor mode. Details are given in the supplementary material. ${ }^{42}$

\section{EXPERIMENTAL METHODS}

5MOI ( $\geq 98 \%)$ was purchased from Carbolution and used without further purification. The experimental setup for the rotationally resolved laser induced fluorescence spectroscopy is described in detail elsewhere. ${ }^{43,44}$ In brief, the laser system consists of a single frequency ring dye laser (Sirah Matisse DS) operated with Rhodamine 6G, pumped with $7 \mathrm{~W}$ of the $514 \mathrm{~nm}$ line of an $\mathrm{Ar}^{+}$-ion laser (Coherent, Sabre $15 \mathrm{DBW}$ ). The dye laser output was coupled into an external folded ring cavity (Spectra Physics Wavetrain) for second harmonic generation. The resulting output power was constant at about $25 \mathrm{~mW}$ during the experiment. The molecular beam was formed by co-expanding $5 \mathrm{MOI}$, heated to $190^{\circ} \mathrm{C}$, and 400 mbar of argon through a $200 \mu \mathrm{m}$ nozzle into the vacuum chamber. The molecular beam machine consists of three differentially pumped vacuum chambers that are linearly connected by skimmers ( $1 \mathrm{~mm}$ and $3 \mathrm{~mm}$, respectively) in order to reduce the Doppler width. The resulting resolution is $18 \mathrm{MHz}$ (FWHM) in this setup. In the third chamber, $360 \mathrm{~mm}$ downstream of the nozzle, the molecular beam crosses the laser beam at a right angle. In the detection volume, which is formed by a concave mirror $(f=25 \mathrm{~mm})$ at a distance of $50 \mathrm{~mm}$ below the crossing of molecular beam and laser beam and a plano-convex lens $(f=50 \mathrm{~mm})$ at a distance of $50 \mathrm{~mm}$ above the molecular beam-laser beam crossing, the Stark plates have been inserted. The fluorescence volume is much less than $1 \mathrm{~mm}^{3}$; hence, effects due to inhomogeneity of the Stark field hardly play any role. The parallel light from the collection optics is then focussed onto the photocathode of a UV enhanced photomultiplier tube (Thorn EMI 9863QB) using a lens of $f=130 \mathrm{~mm}$ at a distance of $70 \mathrm{~mm}$. The Stark plates consist of a parallel pair of electroformed nickel wire grid (18 mesh/mm, $50 \mathrm{~mm}$ diameter) with a transmission of $95 \%$ in the UV. Their mutual distance is roughly $23 \mathrm{~mm}$, symmetrically with respect to the laser beam.

The polarization plane of the incoming laser beam can be rotated by $90^{\circ}$ inside the vacuum chamber by means of an achromatic $\lambda / 2$ plate (Bernhard Halle 240-380 $\mathrm{nm}$ ). The $\lambda / 2$ plate can be pushed in or pulled out from the beamway using a linear motion vacuum feedthrough. Figure 1 shows the imaging optics with the Stark arrangement. The effective distance of the Stark plates was calibrated using Stark spectra of benzonitrile, measured before by Borst et al., ${ }^{1}$ with the exactly known $\mu_{a}$ of the ground state from microwave Stark
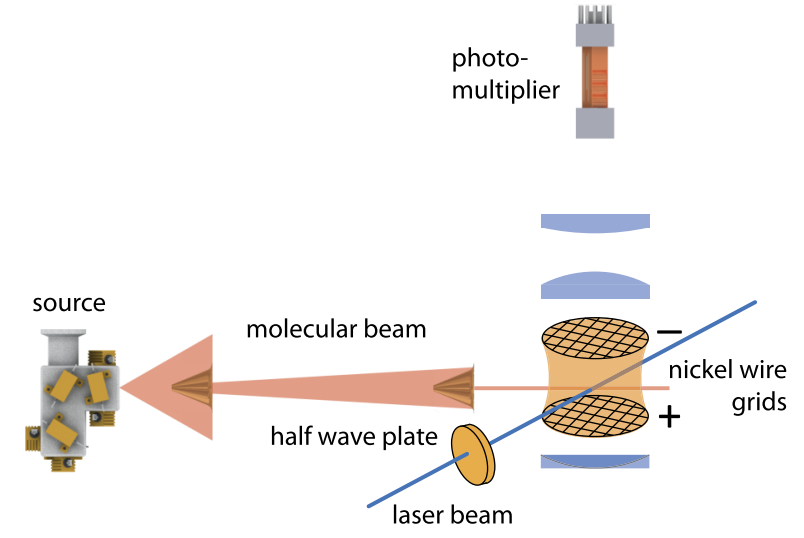

FIG. 1. View of the molecular beam and the imaging optics with the Stark arrangement.

measurements. ${ }^{45}$ The reason for measuring the electronic Stark spectrum of benzonitrile for calibration of the effective Stark plate distance and not the primary standard carbonyl sulfide (OCS) is the fact that OCS does not fluoresce. Nevertheless, through the dipole moments from Ref. 45 this spectrum is directly calibrated with respect to the very accurately known OCS dipole moment. ${ }^{46}$

In order to determine the distance of the Stark plates, we recorded the $S_{1} \leftarrow S_{0}$ origin transition of benzonitrile at $36512.74 \mathrm{~cm}^{-1}$ with a voltage at the Stark plates of $950 \pm 1 \mathrm{~V}$ with parallel field configuration. From all observed bands, we selected 20 transitions which share their excited state level with one of the other transitions so that a list of 10 ground state transitions was created from the combination differences. A stick microwave spectrum with these 10 transitions included was finally fit using a standard assigned frequency fit. Details are given in the supplementary material ${ }^{42}$ and for the standard assigned frequency fit in Appendix B of Ref. 39.

\section{RESULTS}

\section{A. Computational results}

Optimized structures of the lowest three singlet states of the syn- and anti-conformers of 5MOI, calculated at the $\mathrm{CC} 2 / \mathrm{cc}-\mathrm{pVTZ}$ level of theory, can be found in Tables S3-S5 of the supplementary material. ${ }^{42}$ Table I shows the CC2/cc-pVTZ calculated adiabatic excitation energies $v_{0}$ of the lowest two electronically excited singlet states, their oscillator strengths $f$, the angle of the transition dipole moment with the inertial $a$-axis $\theta$, the Cartesian components of the permanent dipole moments $\mu_{a}, \mu_{b}, \mu_{c}$, the total dipole moment $\mu$, and the angle of the permanent dipole moment with the inertial $a$-axis $\theta_{D}$. The angle $\theta_{D}$ will be used in the discussion of the experimental results, since from electronic Stark spectroscopy only the unsigned values of the Cartesian components of the dipole moment can be determined, leading to an indeterminacy of the sign of $\theta_{D}$. This can be removed by comparison to the calculations.

The calculated dipole moment vectors of the syn- and anti-conformer of 5MOI for all three states are shown in Figure 2. The respective centers of nuclear masses and 
TABLE I. Calculated properties of the ground and lowest two electronically excited states of the anti- and syn-conformer of 5MOI at their respective CC2/cc-pVTZ optimized geometries. This includes the zero-point energy corrected adiabatic excitation wavenumber $v_{0}$, the oscillator strength $f$, the angle of the transition dipole moment $\theta$, and the angle of the permanent dipole moment $\theta_{D}$ with the main inertial axis $a$. A positive sign of the angle means a clockwise rotation of the (transition) dipole moment vector onto the $a$-axis. Additionally, the Cartesian components and the permanent dipole moment of the electronic states are given.

\begin{tabular}{lccccccc}
\hline \hline \multirow{2}{*}{ Conformer } & \multicolumn{3}{c}{$a n t i$} & & \multicolumn{3}{c}{ syn } \\
\cline { 2 - 4 } \cline { 6 - 7 } State & $\mathrm{S}_{0}$ & $\mathrm{~S}_{1}$ & $\mathrm{~S}_{2}$ & & $\mathrm{~S}_{0}$ & $\mathrm{~S}_{1}$ & $\mathrm{~S}_{2}$ \\
\hline$\nu_{0} / \mathrm{cm}^{-1}$ & $\ldots$ & 33483 & 37816 & $\ldots$ & 32721 & 37547 \\
$f$ & $\ldots$ & 0.08 & 0.18 & & $\ldots$ & 0.08 & 0.17 \\
$\theta / \mathrm{deg}$ & $\ldots$ & +64 & -1 & $\ldots$ & +64 & -9 \\
$\mu_{a} / \mathrm{D}$ & 1.64 & 0.29 & 5.15 & 1.00 & 0.49 & 4.03 \\
$\mu_{b} / \mathrm{D}$ & 0.07 & 1.05 & 1.27 & 2.35 & 1.70 & 2.03 \\
$\mu_{c} / \mathrm{D}$ & 0.00 & 0.00 & 0.00 & & 0.00 & 0.00 & 0.00 \\
$\mu / \mathrm{D}$ & 1.64 & 1.09 & 5.30 & 2.56 & 1.77 & 4.51 \\
$\theta_{D} / \mathrm{deg}$ & +2 & +75 & +14 & -67 & -106 & -27 \\
\hline \hline
\end{tabular}

nuclear charges are quasi-coincident. While the angles of the transition dipole moments with the main inertial axes and the oscillators strengths $f$ are practically the same for the syn-and anti-conformers of 5MOI, there are large variations in size and even direction of the permanent dipole moments in all three singlet states between the two conformers.
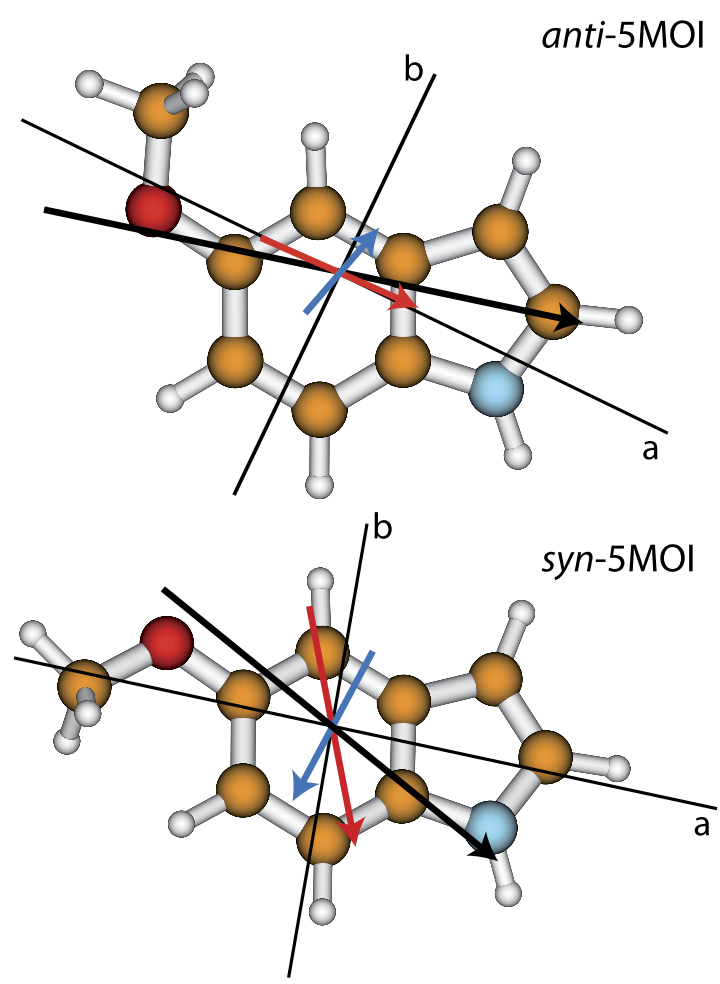

FIG. 2. Calculated dipole moment vectors for the $S_{0}, S_{1}$, and $S_{2}$ states of the anti- and syn-conformer of 5MOI. Red arrows designate the $\mathrm{S}_{0}$-state dipole moments, blue those of the $S_{1}$, and black those of the $S_{2}$-states, respectively. A positive sign of $\theta$ in Table I is defined as clockwise rotation of the dipole moment vector onto the $a$-axis.

\section{B. Experimental results}

Figures 3 and 4 show the rotationally resolved electronic spectra of the electronic origin of anti-5MOI at a field strength of $397.19 \mathrm{~V} / \mathrm{cm}$ measured with almost parallel and perpendicular orientations of the laser light with respect to the static electric field. The simulation in both figures was created by using the best parameters from a CMA-ES fit given in Table II. The uncertainties of the parameters are given in parentheses and are obtained as standard deviations by performing a quantum number assigned fit. This classical fit in which quantum numbers are assigned to each individual transition is described in detail in Appendix B of Ref. 39. In this fit, only transitions are included that would be used in a typical manual assignment of the spectrum, that is, transitions with an intensity larger than $5 \%$ of the maximum intensity and with rotational quantum numbers up to $J=15$ for the calculation of the Stark spectra. A test with larger J-values was performed and showed no visible difference to the $\mathrm{J}=15$ case. A great advantage of the CMA-ES fit is that the analysis of a spectrum created by pure parallel or perpendicular field geometries is also possible as the analysis of a spectrum with a rotated polarization of the laser beam. Moreover, even the ratio between both field configurations can be determined. This is possible because the analysis is not based on quantum numbers but line-shapes. The reason for the deviation of $20 \%$ from a perfect parallel or perpendicular orientation of the laser light with respect to the static electric field can be explained with birefringent effects of the quartz windows due to stress. The fact that the ratio is inversed by inserting the half-wave plate shows that it works correctly and rotates the orientation by $90^{\circ}$.

Figure 5 compares the two Stark spectra of anti-5MOI that were fit together to the dipole moments in both electronic states leading to the dipole moment components of ground and excited states, respectively, presented in Table II. The spectra were fit with the rotational constants, the rotational temperature(s), transition dipole moment orientation, and the band origin wavenumber kept constant at the values from the fit of the field free spectrum. ${ }^{26}$

\section{DISCUSSION}

The permanent dipole moment of anti-5MOI has been determined from electronic Stark spectra with different field configurations. Several fits have been performed on individual spectra and simultaneously on different field configurations. Provision was made in the fitting procedure for the case of mixed selection rules. They result from a deviation of perfectly parallel and perpendicular field geometries. Indeed, the fit showed that the spectrum with parallel geometry is a superposition of $80 \%$ parallel and $20 \%$ perpendicular transitions, while the perpendicular field geometry results in $20 \%$ parallel and $80 \%$ perpendicular transitions. The combined fit increases the precision of the determination of the dipole moment components by a factor of two, as shown in Table III. In this table, the ground and excited state dipole moments and their relative uncertainties from all fits are compared. The relative uncertainties of the combined 

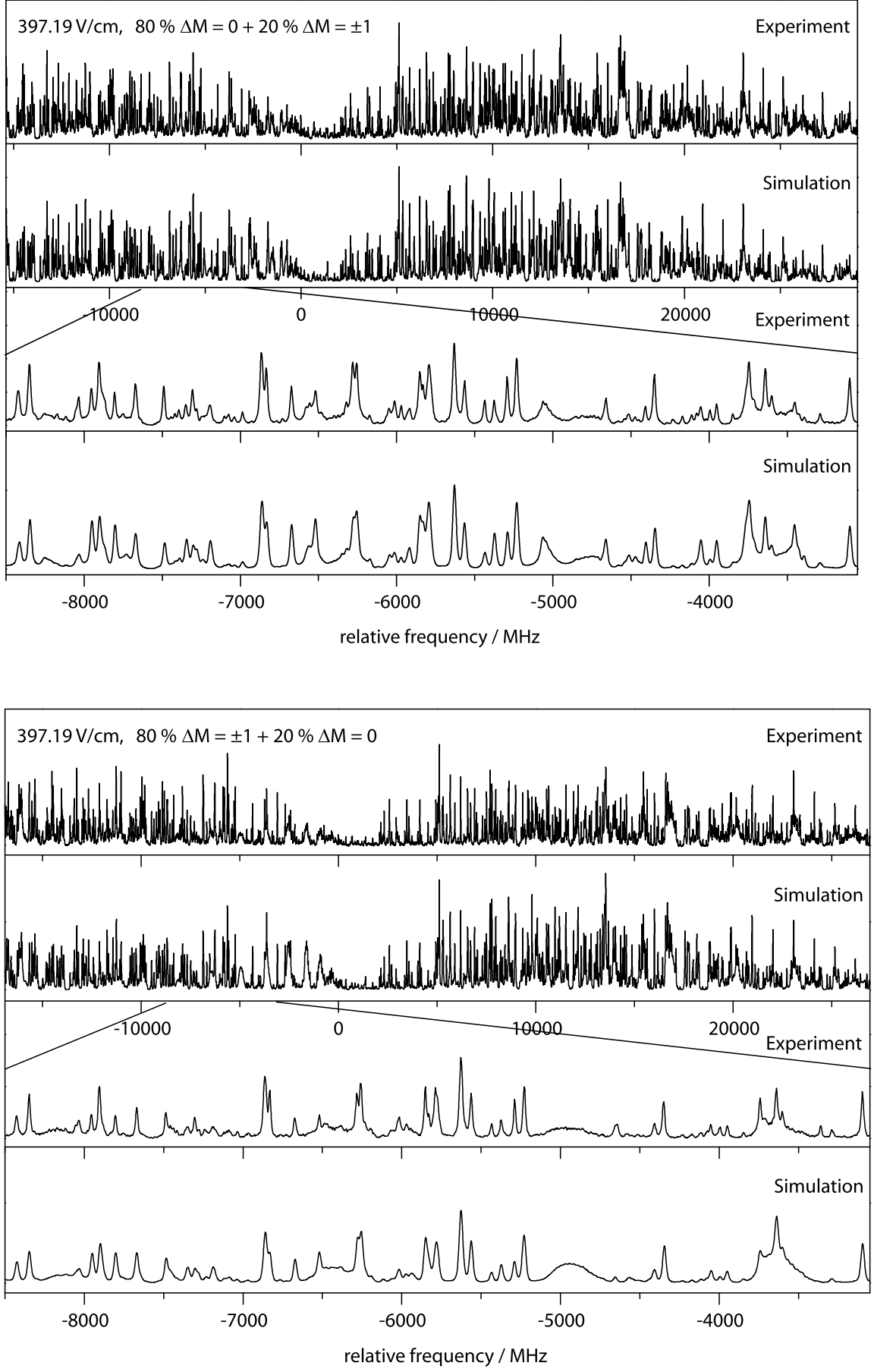

FIG. 3. Rovibronic spectrum of the electronic origin of the anti-conformer of 5-methoxyindole at $397.19 \mathrm{~V} / \mathrm{cm}$ with $80 \%$ transitions of $\Delta M=0$ and $20 \%$ transitions of $\Delta M= \pm 1$ along with a simulation using the best CMA-ES fit parameters given in Table II.
FIG. 4. Rovibronic spectrum of the electronic origin of the anti-conformer of 5-methoxyindole at $397.19 \mathrm{~V} / \mathrm{cm}$ with $80 \%$ transitions of $\Delta M= \pm 1$ and $20 \%$ transitions of $\Delta M=0$ along with a simulation using the best CMA-ES fit parameters given in Table II. fit decrease compared to the individual fits. This can be rationalized, since the consideration of the intensities from both field configurations adds new independent information to the fit. Additionally, the correlation between the parameters is decreased. As the parameter correlation is small in the chosen example, we will not pursue this effect, but it might get importance in cases of stronger parameter correlations. For the sake of clarity, we gave the parameter correlation matrices for three different fits with parallel and perpendicular fits separately and together in the supplementary material. ${ }^{42}$

The experimental results for the dipole moment components in the ground and lowest excited singlet states are compared to the CC2/cc-pVTZ calculated values in Table II. A fair agreement is found both for the absolute values and the components of the dipole moments. Both the components of the transition dipole moment and of the permanent dipole moment suffer from the indeterminacy of the sign of the components, i.e., the sign of the dipole orientation angle with the $a$-axis $\theta_{D}$ and the transition dipole orientation angle $\theta$ are not determined in our experiment. However, from a comparison with the $a b$ initio calculations of the dipole moments, it is clear that the angles $\theta_{D}$ are $+15^{\circ}$ for the $S_{0}$ and $+69^{\circ}$ for the excited state. This means that the dipole moment vector is rotated by $54^{\circ}$ upon excitation to the lowest excited singlet state, cf. the graphical representation of the calculated dipole moments in Figure 2.

Under the assumption that inductive effects are small, the ground state dipole moment vectors of both conformers can 
TABLE II. Experimental and CC2/cc-pVTZ calculated molecular parameters of anti-5MOI determined from the rovibronic Stark spectra of the electronic origin. The rotational constants and the orientation of the transition dipole moment with respect to the inertial $a$-axis $\theta$ were taken from Ref. 26 and kept constant during the fit of the Stark spectra. Changes of the rotational constants are defined as $\Delta B_{g}=B_{g}^{\prime}-B_{g}^{\prime \prime}$, with $B_{g}$ as rotational constants with respect to the inertial axes $g=a, b, c$. Double-primed constants belong to the ground state and single-primed to the excited state. $v_{0}$ is the center frequency of the band, $\Delta v_{\text {Lorentz }}$ the Lorentz contribution to the line width, full width at half-height (FWHM), and $t_{1 / 2}$ the lifetime of the lowest electronically excited state. The angle of the permanent dipole moment with the main inertial axis $a$ is described as $\theta_{D}$. Additionally, the Cartesian components and the permanent dipole moment of the ground $\mu_{i}^{\prime \prime}$ and first electronically excited states $\mu_{i}^{\prime}$ are given. The out-of-plane dipole component $\mu_{c}$ was held fixed at $0.0 \mathrm{D}$ for both electronic states. For the determination of the population of each rovibronic level, the model of $\mathrm{Wu}$ and Levy ${ }^{47}$ is used, where $T_{1}$ and $T_{2}$ are the two temperatures, and $\omega$ is a weighting factor.

\begin{tabular}{|c|c|c|c|}
\hline & \multirow[b]{2}{*}{ Experiment } & \multicolumn{2}{|c|}{ CC2/cc-pVTZ } \\
\hline & & anti-5MOI & syn-5-MOI \\
\hline$A^{\prime \prime} / \mathrm{MHz}$ & $2851.84(15)$ & 2843 & 3408 \\
\hline$B^{\prime \prime} / \mathrm{MHz}$ & $757.92(3)$ & 762 & 707 \\
\hline$C^{\prime \prime} / \mathrm{MHz}$ & $601.33(4)$ & 603 & 588 \\
\hline$\mu_{a}^{\prime \prime} / \mathrm{D}$ & $1.53(1)$ & 1.64 & 1.00 \\
\hline$\mu_{b}^{\prime \prime} / \mathrm{D}$ & $0.42(6)$ & 0.07 & 2.35 \\
\hline$\mu_{c}^{\prime \prime} / \mathrm{D}$ & $0.00(0)$ & 0.00 & 0.00 \\
\hline$\mu^{\prime \prime} / \mathrm{D}$ & $1.59(3)$ & 1.64 & 2.56 \\
\hline$\theta_{D}^{\prime \prime} / \operatorname{deg}$ & $\pm 15(1)$ & +2 & -67 \\
\hline$A^{\prime} / \mathrm{MHz}$ & $2752.50(16)$ & 2738 & 3311 \\
\hline$B^{\prime} / \mathrm{MHz}$ & $759.19(5)$ & 763 & 705 \\
\hline$C^{\prime} / \mathrm{MHz}$ & $597.68(7)$ & 599 & 584 \\
\hline$\Delta A / \mathrm{MHz}$ & $-99.34(22)$ & -105 & -97 \\
\hline$\Delta B / \mathrm{MHz}$ & $1.26(6)$ & +1 & -2 \\
\hline$\Delta C / \mathrm{MHz}$ & $-3.66(8)$ & -4 & -4 \\
\hline$\mu_{a}^{\prime} / \mathrm{D}$ & $0.41(11)$ & 0.29 & 0.49 \\
\hline$\mu_{b}^{\prime} / \mathrm{D}$ & $1.06(2)$ & 1.05 & 1.70 \\
\hline$\mu_{c}^{\prime} / \mathrm{D}$ & $0.00(0)$ & 0.00 & 0.00 \\
\hline$\mu^{\prime} / \mathrm{D}$ & $1.14(6)$ & 1.09 & 1.77 \\
\hline$\theta_{D}^{\prime} / \operatorname{deg}$ & $\pm 69(1)$ & +75 & -106 \\
\hline$\theta /$ deg & $\pm 58.4(5)$ & +64 & +64 \\
\hline$v_{0} / \mathrm{cm}^{-1}$ & $33120.53(2)$ & 33483 & 32721 \\
\hline$\Delta v_{\text {Lorentz }} / \mathrm{MHz}$ & $24(1)$ & $\ldots$ & $\ldots$ \\
\hline$t_{1 / 2} / \mathrm{ns}$ & $6.6(3)$ & $\ldots$ & $\ldots$ \\
\hline$T_{1} / \mathrm{K}$ & 3.77 & $\ldots$ & $\ldots$ \\
\hline$T_{2} / \mathrm{K}$ & 3.02 & $\ldots$ & $\ldots$ \\
\hline$\omega$ & 0.20 & $\ldots$ & $\ldots$ \\
\hline
\end{tabular}

be thought as being composed of the indole dipole moment vector and the dipole moment vector of the methoxy group by vector addition. Inductive effects are consequence of mutual polarization of adjacent bonds, mostly through resonance effects. Since the -M-effect of the methoxy group is small, the assumption seems to be correct in the present case.

The methoxy group dipole moment is obtained from the incremental values of $0.86 \mathrm{D}$ for the $\mathrm{C}-\mathrm{O}$ bond dipole and of $1.16 \mathrm{D}$ for the $\mathrm{H}_{3} \mathrm{C}-\mathrm{O}$ group dipole moment, with an $\mathrm{C}-\mathrm{O}-\mathrm{C}$ angle of $108^{\circ} .{ }^{48}$ Subtracting the so-calculated dipole moment vector of the methoxy group from the experimentally determined anti-5MOI ground state, dipole moment vector should result in the dipole moment vector of unsubstituted indole. The respective vector diagram is shown in the left hand side of Figure 6. It should be pointed out that the anti-5MOI vector has been shifted from the center of charge of the molecule to the methoxy group for the sake of clarity. The dashed-dotted difference vector $\left(\vec{\mu}_{\text {indole }}^{S_{0}}=\vec{\mu}_{5 M O I}^{S_{0}}-\vec{\mu}_{\text {methoxy }}\right)$ has indeed the correct direction of the indole dipole moment, which has been determined from electronic Stark spectroscopy by Pratt and co-workers to be $45.5^{\circ}$ with respect to the inertial $a$-axis in indole. ${ }^{7}$ This inertial axis is shown along with the respective axis of anti-5MOI in Figure 6. The calculated angle from the difference vector to the indole $a$-axis is $45^{\circ}$, the length of the indole dipole vector from Figure 6 is 1.75 $\mathrm{D}$, which is in fair agreement with the absolute experimental value of 1.96 D. ${ }^{7}$ The situation completely changes for the $S_{1}$ state (right hand side of Figure 6). Here, vector subtraction of $\vec{\mu}_{5 M O I}^{S_{1}}$ and $\vec{\mu}_{\text {methoxy }}$ leads to a vector, which is both in direction and size, completely different from the $S_{1}$ state dipole moment vector of indole, reported by Kang et al. ${ }^{7}$ The resulting dipole vector makes an angle of $15^{\circ}$ with the indole $a$-axis, while the experimentally determined angle is $33^{\circ}$, and the absolute value of the dipole moment, represented by this vector, decreases by $1.0 \mathrm{D}$, while the experimental value from Ref. 7 decreases only by $0.1 \mathrm{D}$ upon electronic excitation.

What is the reason for this obvious asymmetry between the additivity rule for dipole increments in the ground and excited state? It is instructive to view two limiting cases for the dipole moment additivity rule: Systems in which the $S_{0}$ dipole increments add vectorially and systems in which this is true for the $S_{1}$ state. The first case is observed, e.g., in 3 -aminophenol. ${ }^{49} 3$-aminophenol is a planar molecule in both electronic states, i.e., the dipole moment lies in the plane of the molecule. The two substituents in meta positions are sufficiently electronically isolated, to prevent interaction of the electrons that are responsible for the dipole moment, leading to an additivity of the dipole moments of aniline and phenol to that of 3-aminophenol. For the $S_{1}$ state of this molecule, the additivity does not hold. Reese et al. ${ }^{49}$ discussed this deviation in light of a different mixing of two electronically excited states for off-axis substituted molecules. Pratt and co-workers have shown that unsymmetrical off-axis substitution in divinylbenzenes leads to a mixing of the $L_{a}$ and $L_{b}$ states. ${ }^{50}$ The other limiting case is found, e.g., for 4-aminobenzonitrile. ${ }^{1}$ In this case, we have a substitution symmetric with respect to the $a$-axis. The dipole moments in the $S_{1}$ states of aniline and benzonitrile add up to the $\mathrm{S}_{1}$ dipole moment of 4-aminobenzonitrile. However, the additivity rule does not work for the $S_{0}$ state. The reason for this unexpected behavior is that aniline and aminobenzonitrile have different degrees of non-planarity in the ground state (benzonitrile is planar). Thus, the heavy atom structure of aniline and aminobenzonitrile is different with respect to the amino group. This applies only to the electronic ground state, since aniline and aminobenzonitrile are planar in the excited state.

5MOI, indole, and the methoxy group have planar symmetry in the electronic ground state. Thus, it is not surprising that we observe additivity for the dipole moment increments in this state. Actually, off-axis substitution violates the original condition of Platt (at least $\mathrm{C}_{2}$-symmetry) for 


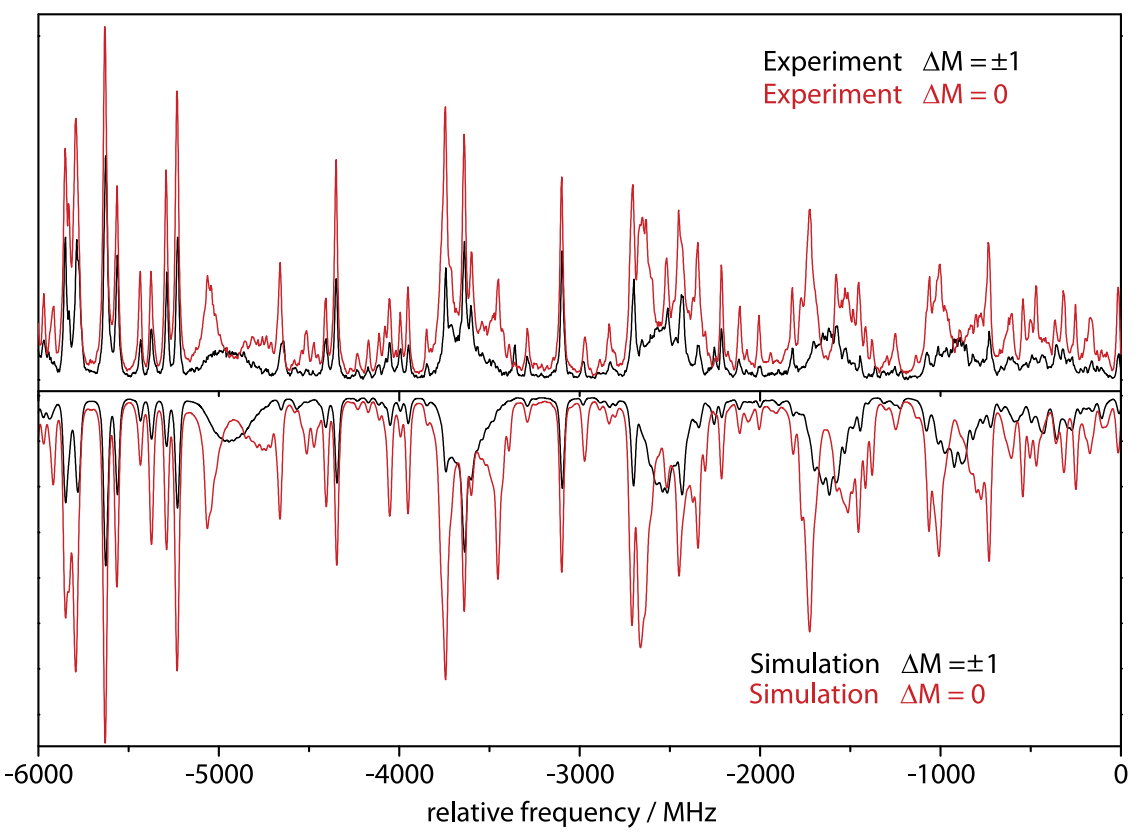

FIG. 5. Experimental Stark spectra of the electronic origin of the anticonformer of 5MOI at electric field strengths of $397.19 \mathrm{~V} / \mathrm{cm}$ with inversed weights of the ratio between a parallel and a perpendicular polarization of the laser beam to the electric field. The red trace belongs to set 1 with an almost parallel $(\Delta M=0)$ and the black trace to set 2 with a nearly perpendicular polarization of the laser beam to the electric field $(\Delta M= \pm 1)$. The lower half of the figure shows the simulated spectra as they are analyzed with the CMA-ES algorithm.

TABLE III. Planar dipole moment components with relative uncertainties in parentheses from various fits of different selection rules at a field strength of $397.19 \mathrm{~V} / \mathrm{cm}$.

\begin{tabular}{lccccc}
\hline \hline & Selection rule & $\mu_{a}^{\prime \prime} / \mathrm{D}$ & $\mu_{b}^{\prime \prime} / \mathrm{D}$ & $\mu_{a}^{\prime} / \mathrm{D}$ & $\mu_{b}^{\prime} / \mathrm{D}$ \\
\hline Set 1 & $80 \% \Delta M=0 / 20 \% \Delta M= \pm 1$ & $1.53(3)$ & $0.39(10)$ & $0.43(15)$ & $1.12(4)$ \\
Set 2 & $80 \% \Delta M= \pm 1 / 20 \% \Delta M=0$ & $1.57(3)$ & $0.35(10)$ & $0.33(20)$ & $1.07(3)$ \\
Set 3 & Set 1 + set 2 & $1.53(1)$ & $0.42(6)$ & $0.41(11)$ & $1.06(2)$ \\
\hline \hline
\end{tabular}

Ground State

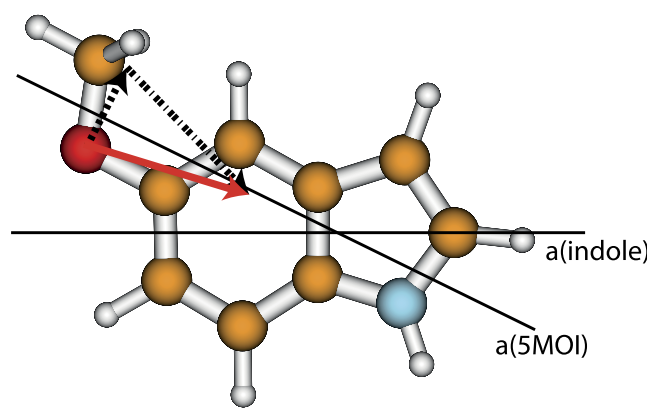

Excited State

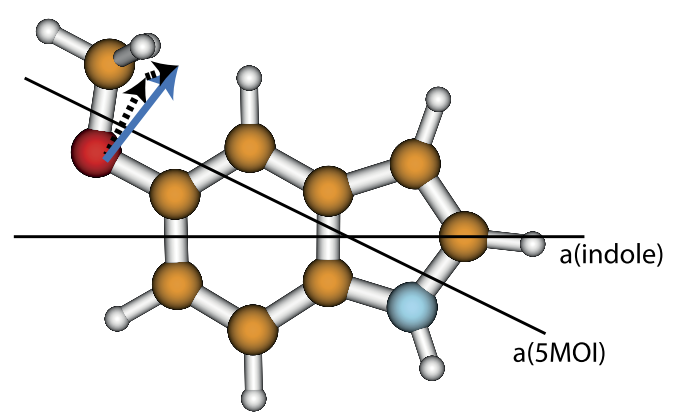

FIG. 6. Left: Vector subtraction of the experimental ground state dipole moment of anti-5MOI (straight red line) and the dipole moment increment of the OCH group (dotted line). The resulting vector is close to the ground state dipole moment of unsubstituted indole (dashed-dotted line). Right: The same diagram for the electronically excited state. The red $\left(\mathrm{S}_{0}\right)$ and blue $\left(\mathrm{S}_{1}\right)$ 5MOI vectors correspond to the experimentally determined vectors in Table II, they are just shifted from the center of charge to the methoxy group for the sake of clarity.

classification as $L_{a}$ or $L_{b}$ states. In our case, the energetic difference between $L_{a}$ and $L_{b}$ states is much smaller in indole than in 5MOI. Therefore, it can be argued that for indole, the excited states mix to a higher extent. Since the $L_{a}$ state has a higher dipole moment than the $L_{b}$ state and a different orientation (cf. Table I), the resulting excited state dipole moment depends strongly on the composition of the excited state. This different amount of $L_{a} / L_{b}$ mixing in indole and $5 \mathrm{MOI}$ is then the reason for the non-additivity of the dipole moments in the excited state. Another reason for the non-additivity of the dipole moments in the excited state might be that $5 \mathrm{MOI}$ shows a considerable participation of charge transfer character in the $S_{1}$ state, which is not present in indole.

In many molecules, it is possible to determine the direction of the transition dipole moment from the frontier orbitals. However, when strong $\sigma$ (inductive) effects govern the electron density distribution after excitation, great care has to be taken, not to overstress the fundamental rules, which are based on electron density effects in the $\pi$-space in case of the frontier orbitals. 


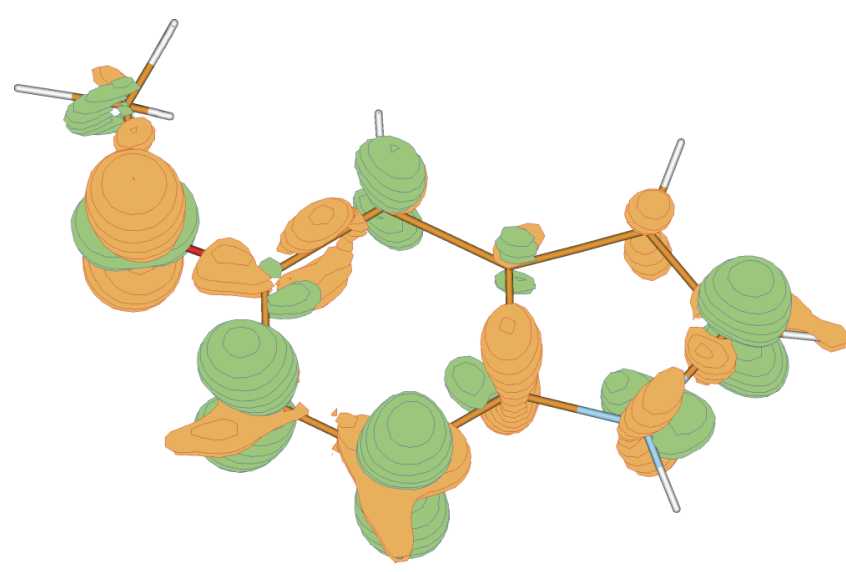

FIG. 7. Electron density difference plot for anti-5MOI. Orange contours indicate regions with loss of electron density and green contours indicate gain of electron density.

Figure 7 shows a plot of the CC2/cc-pVTZ calculated $S_{1}-S_{0}$ electron density differences. Orange contours indicate regions with loss of electron density and green contours indicate gain of electron density. The charge redistribution upon electronic excitation shows a net charge flow from the pyrrole to the benzene ring as in comparable 5-substituted indoles. ${ }^{51}$ The large effect of the methoxy substituent is readily to be seen from Figure 7. While for the methoxy $\pi$ electrons, the electron density decreases (it is shifted into the chromophore), the $\sigma$ density at the methoxy $\mathrm{O}$ atom increases upon electronic excitation. There is an opposite charge density change observed for the $\sigma$ electrons compared to the $\pi$ electrons also for some of the carbon and nitrogen atoms in the indole chromophore. Other atoms show only density changes for the $\pi$ electrons. Since the density changes in the $\sigma$ space are not contained in the frontier orbitals, which all belong to the $\pi$ space, the direction of the transition dipole moment in this molecule cannot be determined in a straightforward manner like for smaller or higher symmetric molecules.

The largest increase of electron density upon electronic excitation happens at $\mathrm{C}(2)$ of the pyrrole ring. In the ground state of indole, it is position $\mathrm{C}(3)$ which has the highest electron density and is the favorite site of electrophilic substitution, due to the possibility of an iminium stabilization. Thus, electrophilic substitution like protonation or alkylation at 2-position in indoles is difficult to achieve. A synthetic route to these products is the transition metal catalyzed 2-alkylation of indoles via $\mathrm{CH}$ activation. From the electron density difference plot, we propose a possible alternative route to 2 -substituted products via a photoinduced activation by excitation to the $S_{1}$ state, cf. Figure 8.

From a natural population analysis using the $\mathrm{CC} 2 / \mathrm{cc}-$ pVTZ wave functions, ${ }^{36}$ we obtain a net charge difference of 0.14 electrons between the pyrrole and the benzene rings upon electronic excitation for the heavy atoms of both rings (excluding the methoxy group contribution). The methoxy group itself donates 0.10 electron charges to the benzene ring upon excitation so that the total effect is only a charge difference of 0.04 electron charges between the rings. Estimating the center of the ring distance between pyrrole and benzene to $2.5 \AA$, we obtain a change of the dipole moment upon electronic excitation of $0.48 \mathrm{D}$, close to the $a b$ initio calculated value of $0.55 \mathrm{D}$ and to the experimental value of $0.45 \mathrm{D}$.

The last topic that we will discuss is the surprising absence of the syn-conformer in the molecular beam studies published until now. Since there is nothing special about this conformer, while for similar substituted indoles, both rotamers are observed experimentally (e.g., 5-hydroxyindole $(5 \mathrm{HOI})^{52}$ and 6-methoxyindole ${ }^{53}$ ), the most plausible explanation for the absence of syn-5MOI in the molecular beam spectrum is given by Florio et al., namely, that "the preexpansion population in syn-oriented 5MOI can be converted into the anti-conformer via collisions with the buffer gas in the expansion." 27 This happens efficiently due to the low barrier to isomerization of about $1.4 \mathrm{kcal} / \mathrm{mol} .{ }^{27} \mathrm{We}$ calculated the energy difference of the syn- and anti-conformers of 5MOI at the CC2/cc-pVTZ level of theory to be $409 \mathrm{~cm}^{-1}$ (including zero-point energy correction) with the anti-conformer being the more stable one, and the barrier between them to $567 \mathrm{~cm}^{-1}$, cf. Figure 9 . This is about half of the barrier in 5HOI $\left(973 \mathrm{~cm}^{-1}\right)$ and at the same time, the relative stability of anti- vs. syn-conformer increases from $155 \mathrm{~cm}^{-1}$ for $5 \mathrm{HOI}$ to $409 \mathrm{~cm}^{-1}$ for $5 \mathrm{MOI}$. The combined effect of the larger stabilization of the anti-minimum of 5MOI with respect to $5 \mathrm{HOI}$ and the much lower barrier separating the 5MOI conformers is most probably the reason for the dominance of the syn-conformer in the 5HOI spectrum and its absence in the 5MOI spectrum.<smiles>C[C@@H]1Cc2ccccc2N1</smiles><smiles>Cc1cc2c([I-])cccc2[nH]1</smiles>

FIG. 8. Protonation at the region of highest electron density at $\mathrm{C}(3)$ for the ground state with a subsequent stabilization of the iminium ion expressed by two tautomeric forms (upper half of the figure) and at $\mathrm{C}(2)$ for the excited state (lower half of the figure). 


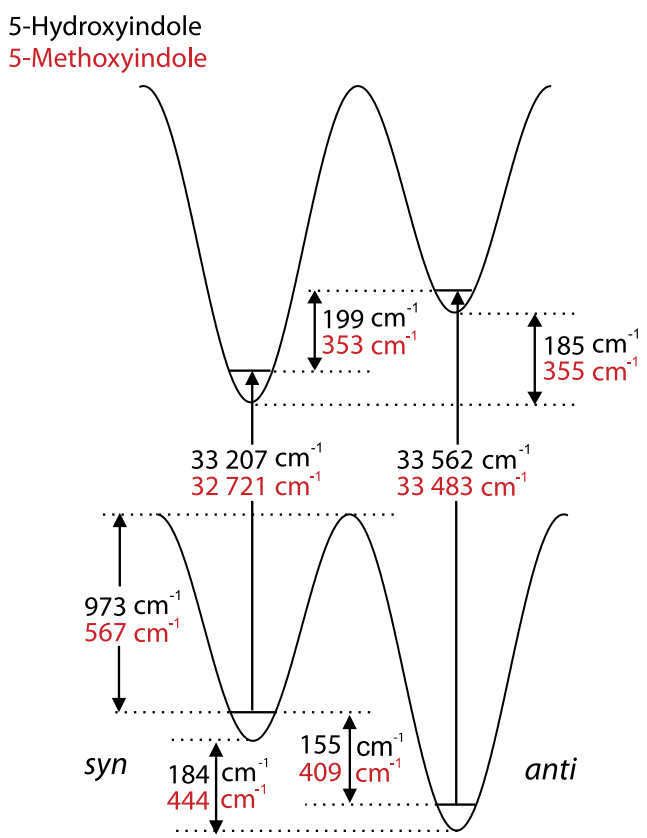

FIG. 9. CC2/cc-pVTZ calculated relative energies of the syn- and anticonformers of $5 \mathrm{MOI}$ (red numbers) and 5HOI (black numbers), their $\mathrm{S}_{0}-\mathrm{S}_{1}$ excitation energies, and barriers separating the minima of the conformers.

\section{CONCLUSIONS}

Comparison of the excited state dipole moment to that of the ground state for anti-5MOI shows that the dipole moment, which is oriented nearly along the inertial $a$-axis in the ground state, is rotated by $-54^{\circ}$ anticlockwise. This rotation is much larger than the respective rotation of the dipole moment upon excitation in bare indole of $-12^{\circ}{ }^{7}$ Furthermore, the absolute value of $\mu$ decreases from $1.59 \mathrm{D}$ in the ground state to 1.14 $\mathrm{D}$ in the excited state of anti-5MOI $(\Delta \mu=-0.45 \mathrm{D})$, larger than in indole $(\Delta \mu=-0.11 \mathrm{D})$. The CC2/cc-pVTZ calculated values of $-73^{\circ}$ for $\theta_{D}$ and of $-0.55 \mathrm{D}$ for $\Delta \mu$ of anti-5MOI match fairly well.

For the electronic ground state, the dipole moment vector of anti-5MOI can be constructed from a vector addition of the methoxy group and the indole dipole moment vectors with good precision. This procedure completely fails for the lowest excited singlet state. Electronic excitation leads to a changed electron density in the chromophore, which is partially accompanied by an electron density shift from or to the substituent. Depending on the acceptor or donor properties of the substituents and even more on the electronic nature (mesomeric or inductive substituents), the concept of vector addition of bond or group dipole moments completely fails for excited states.

Finally, we could show that the precision of the determination of dipole moments of the lowest two singlet states of 5MOI from optical Stark measurement in rovibronically resolved spectra can be improved by a simultaneous fit of two spectra with both field configurations leading to different $\Delta M$ selection rules. Comparison to the results of $\mathrm{CC} 2 / \mathrm{cc}-$ pVTZ calculations shows a fair agreement for the absolute experimental value not only of the total dipole moment in the $S_{0}$ and the $S_{1}$ states but also for the respective Cartesian components of the dipole moments.
One might ask whether a field configuration with $45^{\circ}$ between the static electric field and the exciting laser field would not lead to the same results in a single spectrum. We tested this technique and found that the spectral congestion due to the existence of 50\% parallel and 50\% perpendicular transitions in one spectrum deteriorates the fit of the dipole moments considerably. For the future, we plan to extend the experiment with a $\lambda / 4$ wave plate. Using right and left circular polarized light, the $\Delta M= \pm 1$ transitions can be broken down to $\Delta M=+1$ and $\Delta M=-1$ transitions, therefore reducing the complexity and congestion of dense Stark spectra of larger molecules. These spectra can then again be fitted along with spectra from different field configurations.

Spectra taken at lower field strength show a decreased precision for the dipole moment determination. Since for 5MOI, the chosen field strength is clearly still not sufficient for pulling out all $M$-states from transitions up to $J=15$, which are populated under our molecular beam conditions. Still higher field strengths will enable us to increase the precision further, especially for the case of small absolute dipole moments and/or small changes of the dipole moment upon electronic excitation. Currently, we are implementing a new high voltage source which will allow to reach field strengths up to $10 \mathrm{kV} / \mathrm{cm}$. This is achieved by increasing the applied voltage and decreasing the plate separation at the same time. Effects due to field inhomogeneities are minimized by a new plate configuration with a grounded ring electrode around the existing plate setup. Using this new setup, the precision with which the dipole moments can be determined will be increased, while at the same time, much smaller dipole moments can still be measured. From simulations for a field strength of $10 \mathrm{kV} / \mathrm{cm}$, we deduce that an absolute dipole moment down to $0.01 \mathrm{D}$ for one state or a difference down to $0.01 \mathrm{D}$ between two states can still be measured. The determination of small absolute dipole moments along with small changes upon vibrational excitation using high field strengths of over $50 \mathrm{kV} / \mathrm{cm}$ has been shown for the propargyl radical in helium droplets by Küpper et al. ${ }^{54}$

\section{ACKNOWLEDGMENTS}

We gratefully acknowledge the help of David Plusquellic for making available the source of the Stark program for an asymmetric rotor molecule which was originally developed by David Borst in the group of David Pratt. Michael Schmitt thanks the Deutsche Forschungsgemeinschaft for financial support of this work (SCHM No. 1043/12-1). Computational support and infrastructure was provided by the "Center for Information and Media Technology" (ZIM) at the HeinrichHeine-University Düsseldorf (Germany).

${ }^{1}$ D. R. Borst, T. M. Korter, and D. W. Pratt, Chem. Phys. Lett. 350, 485 (2001).

${ }^{2}$ W. G. J. Hol, P. T. V. Duijnen, and H. J. C. Berendsen, Nature 273, 443 (1978).

${ }^{3}$ D. R. Ripoll, J. A. Vila, and H. A. Scheraga, Proc. Natl. Acad. Sci. U. S. A. 102, 7559 (2010).

${ }^{4}$ J. R. Platt, J. Chem. Phys. 17, 484 (1949).

${ }^{5}$ G. Weber, Biochem. J. 75, 335 (1960).

${ }^{6}$ A. L. Sobolewski and W. Domcke, Chem. Phys. Lett. 315, 293 (1999). 
${ }^{7}$ C. Kang, T. M. Korter, and D. W. Pratt, J. Chem. Phys. 122, 174301 (2005).

${ }^{8} \mathrm{~W}$. Liptay, "Dipole moments and polarizabilities of molecules in excited electronic states," in Excited States, edited by E. Lim (Academic Press, New York, 1974), Vol. I, Chap. 4, pp. 129-229.

${ }^{9}$ P. Suppan, J. Photochem. Photobiol., A 50, 293 (1990).

${ }^{10} \mathrm{~T}$. Abe and I. Iweibo, Bull. Chem. Soc. Jpn. 58, 3415 (1984).

${ }^{11}$ J. R. Lombardi, J. Phys. Chem. A 102, 2817 (1998).

${ }^{12}$ J. R. Lombardi, J. Phys. Chem. A 103, 6335 (1999).

${ }^{13}$ A. Kawski, B. Kuklinski, and P. Bojarski, Chem. Phys. Lett. 415, 251 (2005).

${ }^{14}$ A. Kawski, B. Kuklinski, and P. Bojarski, Chem. Phys. Lett. 419, 309 (2006).

${ }^{15}$ M. Okruss and B. R. A. Hese, Chem. Phys. Lett. 220, 286 (1994).

${ }^{16}$ T. M. Korter, D. R. Borst, C. J. Butler, and D. W. Pratt, J. Am. Chem. Soc. 123, 96 (2001).

${ }^{17}$ P. J. Brucat and R. N. Zare, Mol. Phys. 55, 277 (1985).

${ }^{18}$ P. Schmidt, H. Bitto, and J. R. Huber, J. Chem. Phys. 88, 696 (1988).

${ }^{19}$ N. Ohta and T. Tanaka, J. Chem. Phys. 99, 3312 (1993).

${ }^{20}$ M. Martinaud and A. Kadiri, Chem. Phys. 28, 473 (1978).

${ }^{21}$ M. R. Eftink, L. A. Selvidge, P. R. Callis, and A. A. Rehms, J. Phys. Chem. 94, 3469 (1990).

${ }^{22}$ Y. Yamamoto and J. Tanaka, Bull. Chem. Soc. Jpn. 45, 1362 (1972).

${ }^{23}$ B. Albinsson and B. Nordén, J. Phys. Chem. 96, 6204 (1992).

${ }^{24}$ J. W. Hager, D. R. Demmer, and S. C. Wallace, J. Phys. Chem. 91, 5513 (1984).

${ }^{25}$ Y. Huang and M. Sulkes, J. Phys. Chem. 100, 16479 (1996).

${ }^{26}$ C. Brand, O. Oeltermann, D. W. Pratt, R. Weinkauf, W. L. Meerts, W. van der Zande, K. Kleinermanns, and M. Schmitt, J. Chem. Phys. 133, 024303 (2010).

${ }^{27}$ G. M. Florio, R. A. Christie, K. D. Jordan, and T. S. Zwier, J. Am. Chem. Soc. 124, 10236 (2002).

${ }^{28}$ C. Hättig and F. Weigend, J. Chem. Phys. 113, 5154 (2000).

${ }^{29}$ C. Hättig and A. Köhn, J. Chem. Phys. 117, 6939 (2002).

${ }^{30}$ C. Hättig, J. Chem. Phys. 118, 7751 (2002).

${ }^{31}$ R. Ahlrichs, M. Bär, M. Häser, H. Horn, and C. Kölmel, Chem. Phys. Lett. 162, 165 (1989).

${ }^{32}$ J. T. H. Dunning, J. Chem. Phys. 90, 1007 (1989).

${ }^{33}$ P. Deglmann, F. Furche, and R. Ahlrichs, Chem. Phys. Lett. 362, 511 (2002).
${ }^{34}$ Turbomole v6.5 2013, a development of University of Karlsruhe and Forschungszentrum Karlsruhe GmbH, 1989-2007, TURBOMOLE GmbH, since 2007, available from http://www.turbomole.com.

${ }^{35}$ T. Helgaker, Chem. Phys. Lett. 182, 503 (1991).

${ }^{36}$ A. E. Reed, R. B. Weinstock, and F. Weinhold, J. Chem. Phys. 83, 735 (1985).

${ }^{37}$ W. L. Meerts, M. Schmitt, and G. Groenenboom, Can. J. Chem. 82, 804 (2004).

${ }^{38}$ W. L. Meerts and M. Schmitt, Phys. Scr. 73, C47 (2005).

${ }^{39}$ W. L. Meerts and M. Schmitt, Int. Rev. Phys. Chem. 25, 353 (2006).

${ }^{40} \mathrm{M}$. Schmitt and W. L. Meerts, in Handbook of High Resolution Spectroscopy, edited by M. Quack and F. Merkt (John Wiley and Sons, 2011), ISBN: 9780-470-06653-9.

${ }^{41}$ D. R. Borst, Ph.D. thesis, University of Pittsburgh, Pittsburgh, 2001.

${ }^{42}$ See supplementary material at http://dx.doi.org/10.1063/1.4940689 for detailed information about the calibration method, the matrix elements of the rigid rotor Stark Hamiltonian, the parameter correlation matrix of the dipole moment components, and the optimized Cartesian coordinates of anti-5MOI for the lowest three singlet states resulting from $\mathrm{CC} 2 / \mathrm{cc}-\mathrm{pVTZ}$ calculations.

${ }^{43}$ M. Schmitt, "Spektroskopische untersuchungen an wasserstoffbrückenbindungen," Habilitation, Heinrich-Heine-Universität, MathematischNaturwissenschaftliche Fakultät, Düsseldorf, 2000.

${ }^{44}$ M. Schmitt, J. Küpper, D. Spangenberg, and A. Westphal, Chem. Phys. 254, 349 (2000).

${ }^{45}$ K. Wohlfart, M. Schnell, J. U. Grabow, and J. Küpper, J. Mol. Spectrosc. 247, 119 (2014).

${ }^{46}$ J. Reinartz and A. Dymanus, Chem. Phys. Lett. 24, 346 (1974).

${ }^{47}$ Y. R. Wu and D. H. Levy, J. Chem. Phys. 91, 5278 (1989).

${ }^{48}$ T. Tripathy and B. R. De, J. Phys. Sci. 12, 155 (2008); available at http://14. 139.211.206:8080/jspui/handle/123456789/3.

${ }^{49}$ J. A. Reese, T. V. Nguyen, T. M. Korter, and D. W. Pratt, J. Am. Chem. Soc. 126, 11387 (2004).

${ }^{50}$ T. V. Nguyen, J. W. Ribblett, and D. W. Pratt, Chem. Phys. 283, 279 (2002).

${ }^{51}$ C. Brand, O. Oeltermann, M. Wilke, J. Tatchen, and M. Schmitt, ChemPhysChem 13, 3134 (2012).

${ }^{52}$ O. Oeltermann, C. Brand, M. Wilke, and M. Schmitt, J. Phys. Chem. A 116, 7873 (2012).

${ }^{53}$ C. Brand, O. Oeltermann, M. Wilke, and M. Schmitt, J. Chem. Phys. 138, 024321 (2013).

54J. Küpper, J. M. Merritt, and R. E. Miller, J. Chem. Phys. 117, 647 (2002). 